UC-34 Physics and Mathematics (TID-4500, 15th Ed.)

Contract No. W-7405-eng-92

THE DIFFUSION OF RADIOACTIVE FISSION PRODUCTS

FROM POROUS FUEL ELEMENTS

by

Stephen D. Beck

April 18, 1960

BATTELLE MEMORIAL INSTITUTE

$505 \mathrm{King}$ Avenue

Columbus 1, Ohio 


\section{DISCLAIMER}

This report was prepared as an account of work sponsored by an agency of the United States Government. Neither the United States Government nor any agency Thereof, nor any of their employees, makes any warranty, express or implied, or assumes any legal liability or responsibility for the accuracy, completeness, or usefulness of any information, apparatus, product, or process disclosed, or represents that its use would not infringe privately owned rights. Reference herein to any specific commercial product, process, or service by trade name, trademark, manufacturer, or otherwise does not necessarily constitute or imply its endorsement, recommendation, or favoring by the United States Government or any agency thereof. The views and opinions of authors expressed herein do not necessarily state or reflect those of the United States Government or any agency thereof. 


\section{DISCLAIMER}

Portions of this document may be illegible in electronic image products. Images are produced from the best available original document. 
TABLE OF CONTENTS

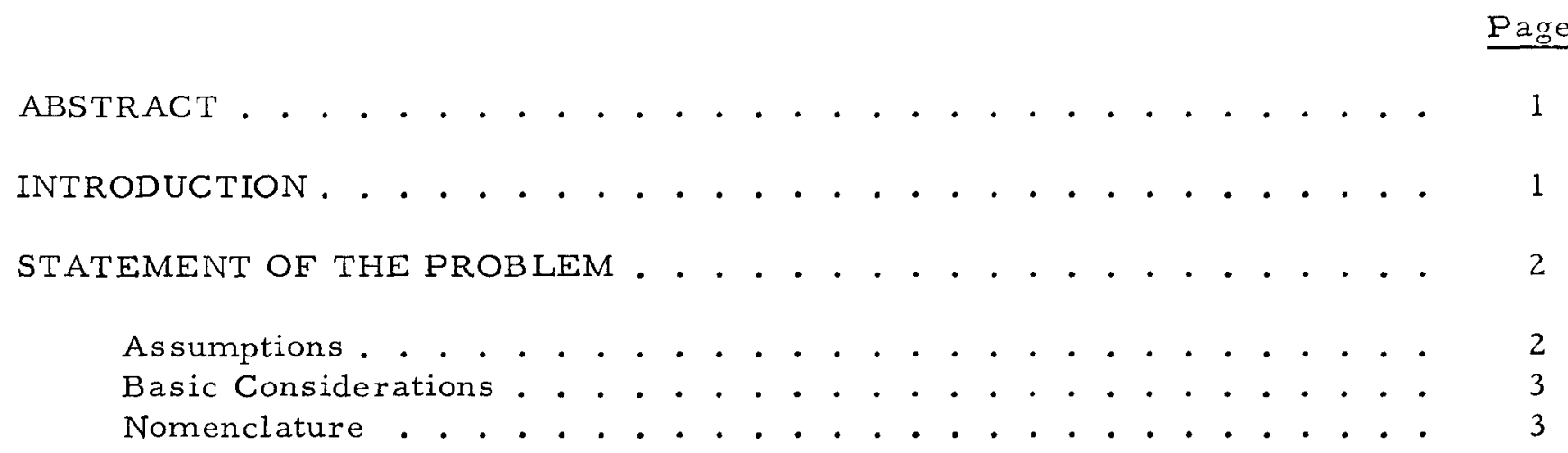

ANALYSIS OF FISSION-PRODUCT RELEASE . . . . . . . . . . . . . . . . 4

Diffusion of Fission Products .................. . 4

Short-Time Approximations . . . . . . . . . . . . . . 7

APPLICATION TO REACTOR CONDITIONS . . . . . . . . . . . . . . . 8

Numerical Evaluation of Fission-Product Release . . . . . . . . . 8

Interpretation of Data . . . . . . . . . . . . . . . . 17

CONCLUSIONS . . . . . . . . . . . . . . . . . . . . 21

ACKNOWLEDGMENTS . . . . . . . . . . . . . . . . . . . 21

REFERENCES . . . . . . . . . . . . . . . . . . . . . . . 22

APPENDIX

DERIVATION OF SHORT-TIME-RELEASE FORMULAS . . . . . . . . . . A- 


\title{
THE DIFFUSION OF RADIOACTIVE FISSIC IV PRODUCTS FROM POROUS FUEL ELEMENTS
}

\author{
Stephen D. Beck
}

\begin{abstract}
The release of fission products from porous fuel elements during irradiation may be largely a diffusion process. An equivalent-sphere hypothesis has been proposed to provide a model by which the diffusion can be analyzed.
\end{abstract}

The equations of diffusion have been previously solved for in-pile conditions. The slow convergence of the formulas makes the previous solutions awkward. In the present investigation alternate formulas were derived which are more suitable under certain circumstances.

Tables have been prepared from which release rates and accumulations may be evaluated for prescribed conditions. The application of the analysis to the interpretation of release data is explained.

\section{INTRODUCTION}

An important problem of reactor design is that of estimating the hazard due to radioactive fission products (especially rare gases) released from the fuel element. Several mechanisms, such as recoil, evaporation, and diffusion, for example, may contribute to the total release. The relative contributions made by the individual mechanisms are not well established, and may depend on several factors. These may include the nature of the fuel material, the design of the fuel element, and the operating temperatures.

We shall concern ourselves in this report with one of the mechanisms, diffusion. More specifically, we will consider the diffusion from porous fuel materials, in which the porosity is essentially interconnected. We assume that the fission products diffuse through the solid matrix of the fuel element into the pores, from which they immediately escape to the exterior of the element.

The analysis is based on the equivalent-sphere model, in which the fuel material is assumed to be composed of a set of spherical particles of equal size. These hypothetical particles do not necessarily resemble the physical structure of the fuel material. We expect, however, that the radius of the equivalent sphere is essentially that for which the surface area-to-volume ratio is the same as the surface area-to-volume ratio of the bulk fuel element. The surface area of the fuel element includes the contribution of the interconnected porosity, and this contribution ordinarily is much larger than the nominal surface of the fuel element.

The idea of an equivalent particle was apparently first proposed by Cubicciotti(1). His particle was an infinitely long cylinder, chosen because of its resemblance to the structure of uranium carbide. Booth $(2,3)$ suggested the use of the equivalent sphere to represent the fuel structure. He found the solutions to the appropriate diffusion equations for stable isotopes for both postirradiation and in-pile situations. Booth(4) also considered the system in which the species was radioactive. However, for this case he (1) References at end of text. 
reported the solution only for steady state, that is, when the behavior is no longer time dependent. Eichenberg, et al., (5) reported the general solution for the accumulated release of an unstable isotope. However, the solution involves an infinite series which becomes too slowly convergent for practical use at short times, although it is theoretically exact for all values of time. Attempis to evaluate the solution, even by machine methods (6), have led to results of doubtful accuracy at very short times due to the extremely slow convergence of the series.

In this report, we are concerned with examining the equivalent-sphere model in somewhat greater detail. In particular, we show how the general formulas for in-pile release of unstable isotopes may be modified to give formulas useful for short times. We use these short-time formulas along with the previously established long-time formulas to prepare tables from which one may determine the rate of release or the accumulation of released activity for specified operating conditions.

It should be pointed out, moreover, that our investigation is essentially concerned with diffusion from a sphere. It does not matter whether this sphere is a hypothetical, so-called equivalent sphere, or if it is an actual sphere from which diffusion release is occurring.

It is also pointed out that the results strictly apply only to the problem which is described in the next section. The justification for applying these results to a real reactor problem must be carefully considered. Our results are useful to the extent that the idealized problem fits the real situation.

\section{STATEMENT OF THE PROBLEM}

\section{Assumptions}

A homogeneous sphere, made up of a fissile material and initially free of a given isotope, is irradiated at a constant burnup rate and a constant temperature. The isotope is formed directly from fission and is produced at a rate which is constant with both position and time. The surface of the sphere remains free of concentration. The isotope diffuses through the sphere according to the laws of Fickian diffusion. The isotope decays by the usual radioactive decay, with the same decay constant governing its behavior before and after release from the sphere. Each sphere is the same as the others.

The assumptions imply that certain phenomena are neglected. These include the contributions of recoil and evaporation, the effect of a precursor on the release, and the effect of neutron absorption on the decay rate. The equivalent-sphere concept implies that we neglect the effects of closed porosity and the time required to traverse the open porosity to the exterior of the fuel element. In addition, the actual structure of the fuel element is replaced with an idealized structure. 


\section{Basic Considerations}

The diffusion equation is solved to give the concentration of a given isotope in the equivalent sphere. The concentration generally depends on position and time. Knowing the concentration permits us to calculate the rate of release and the accumulation from the sphere. The accumulation is defined to be the number of atoms of the isotope which have been released and which have not yet decayed. It is convenient to refer to both the release rate and the accumulation as that from a unit volume of sphere, which is equivalent to saying from a unit volume of the fuel element.

We choose to work in units of atoms, centimeters, and seconds. The choice, however, is completely arbitrary and the results apply generally to any consistent set of units.

A word about notation is in order. Throughout the report an integer in parentheses shall refer to a power of ten. Thus, $(-3)=10^{-3} ; 2.0(4)=2.0 \times 10^{4}$; etc.

\section{Nomenclature}

$$
\begin{aligned}
& \mathrm{C}=\text { concentration in the sphere (atoms per } \mathrm{cm}^{3} \text { ) } \\
& D=\text { diffusion coefficient }\left(\mathrm{cm}^{2}\right. \text { per sec) } \\
& \mathrm{B}=\text { production rate }\left[\text { atoms } /(\mathrm{sec})\left(\mathrm{cm}^{3}\right)\right] \\
& \lambda=\text { decay coefficient }\left(\sec ^{-1}\right) \\
& t=\operatorname{time}(\mathrm{sec}) \\
& r=\text { radial coordinate in the sphere }(\mathrm{cm}) \\
& a=\text { radius of the sphere }(\mathrm{cm}) \\
& \mathrm{R}=\text { rate of release from unit volume of sphere }\left[\text { atoms } /(\mathrm{sec})\left(\mathrm{cm}^{3}\right)\right] \\
& \mathrm{N}=\text { accumulation of undecayed, released atoms from a unit volume of } \\
& \text { sphere (atoms per } \mathrm{cm}^{3} \text { ) } \\
& D^{\prime}=D / a^{2}\left(\sec ^{-1}\right) \\
& \mathrm{H}=\mathrm{R} / \mathrm{B} \\
& \mathrm{G}=\mathrm{N} \lambda / \mathrm{B} \\
& \tilde{\mathrm{F}}=\tilde{\mathrm{N}} / \mathrm{Bt} \\
& \mu=\lambda \mathrm{a}^{2} / \mathrm{D} \\
& \tau=\mathrm{Dt} / \mathrm{a}^{2}
\end{aligned}
$$


The tilde $(\sim)$ over a symbol corresponds to a stable isotope. The subscript infinity $(\propto)$ refers to the steady-state or equilibrium value of a variable.

\section{ANALYSIS OF FISSION-PRODUCT RELEASE}

\section{Diffusion of Fission Products}

The solution of the release problem is outlined, although the final result has been given previously in the literature(5). This is done in order that we may have intermediate results which are of interest.

The diffusion equation for the concentration of a given isotope in the sphere takes into account the production and decay of the species. The equation, then, is $(4,7)$

$$
\frac{\partial C}{\partial t}=\frac{D}{r} \frac{\partial^{2}(r C)}{\partial r^{2}}+B-\lambda C
$$

with boundary and initial conditions,

$$
C(a, t)=C(r, 0)=0 .
$$

If we define $U$ such that

$$
C=\frac{1}{r}\left[U \exp (-\lambda t)+\frac{B}{\lambda}\left(r-\frac{a \sinh r \sqrt{\lambda / D}}{\sinh a \sqrt{\lambda / D}}\right)\right],
$$

we may replace Equation (1) by

$$
\frac{\partial U}{\partial t}=D \frac{\partial^{2} U}{\partial r^{2}}
$$

and the boundary and initial conditions by

$$
\mathrm{U}(0, \mathrm{t})=\mathrm{U}(\mathrm{a}, \mathrm{t})=0 \text {, }
$$

and

$$
U(r, 0)=\frac{B}{\lambda}\left(\frac{a \sinh r \sqrt{\lambda / D}}{\sinh a \sqrt{\lambda / D}}-r\right) .
$$

The first condition (5) follows from the requirement of finite concentration at $r=0$.

The solution to Equation (4) is readily found using Fourier series ${ }^{(8)}$. Finally, the concentration is found by again making the substitution (3). We find then, that

$C=\frac{B}{\lambda r}\left[r-\frac{a \sinh r \sqrt{\lambda / D}}{\sinh a \sqrt{\lambda / D}}+\frac{2 \lambda a^{3} \exp (-\lambda t)}{D \pi} \sum_{n=1}^{\infty} \frac{(-1)^{n} \sin (n \pi r / a) \exp \left(-n^{2} \pi^{2} D t / a^{2}\right)}{n\left(n^{2} \pi^{2}+\lambda a^{2} / D\right)}\right]$. 
The rate of release is found from the concentration gradient at the outer surface. Defining the release in terms of that from a unit volume of the solid, we use the relationship

$$
\mathrm{R}=-\frac{3 \mathrm{D}}{\mathrm{a}}\left(\frac{\partial \mathrm{C}}{\partial \mathrm{r}}\right)_{\mathrm{r}=\mathrm{a}} .
$$

It is convenient to express the release rate nondimensionally, permitting us to write

$$
\mathrm{H}=3\left(\frac{1}{\sqrt{\mu}} \operatorname{coth} \sqrt{\mu}-\frac{1}{\mu}\right)-6 \exp (-\mu \tau) \sum_{\mathrm{n}=1}^{\infty} \frac{\exp \left(-\mathrm{n}^{2} \pi^{2} \tau\right)}{\mathrm{n}^{2} \pi^{2}+\mu} .
$$

$H$ is also the ratio of the total rate at which atoms of an isotope are being released to the total rate at which they are being produced, and may be thought of as the fractional release rate. The release rate will approach an equilibrium value, corresponding to infinite time, for which

$$
\mathrm{H}_{\infty}=3[(1 / \sqrt{\mu}) \operatorname{coth} \sqrt{\mu}-1 / \mu] \text {. }
$$

A special case of Equation (9) is that for which there is no decay, i. e. , $\mu=0$. For this limiting case,

$$
\tilde{H}=1-\left(6 / \pi^{2}\right) \sum_{n=1}^{\infty}\left(1 / n^{2}\right) \exp \left(-n^{2} \pi^{2} \tau\right)
$$

This fractional release rate at equilibrium becomes unity.

We may calculate the accumulation of an isotope by considering the rate at which it is released and the rate at which it decays. Again we refer to the contribution from a unit volume of the solid. The accumulation $N=N(t)$ satisfies the equation

$$
\frac{\mathrm{dN}}{\mathrm{dt}}=\mathrm{R}-\lambda \mathrm{N}
$$

where $\mathrm{N}$ is initially zero. This may be restated in terms of the dimensionless variables of the system as

$$
\mathrm{G}=\exp (-\mu \tau) \int_{0}^{\tau} \mu \exp (\mu \tau) \mathrm{HI} \mathrm{d} \tau
$$

It is found then, that

$\mathrm{G}=3\left(\frac{1}{\sqrt{\mu}} \operatorname{coth} \sqrt{\mu}-\frac{1}{\mu}\right)[1-\exp (-\mu \tau)]-\frac{6 \mu \exp (-\mu \tau)}{\pi^{2}} \sum_{n=1}^{\infty} \frac{1-\exp \left(-\mathrm{n}^{2} \pi^{2} \tau\right)}{\mathrm{n}^{2}\left(\mathrm{n}^{2} \pi^{2}+\mu\right)}$.

This is equivalent to the formula which has been previously reported by Eichenberg, et al. (5) As $\tau$ becomes infinite, $G$ approaches the equilibrium value,

$$
\mathrm{G}_{\infty}=3[(1 / \sqrt{\mu}) \operatorname{coth} \sqrt{\mu}-1 / \mu],
$$


which is the result given previously by Booth(4). We notice trom Equations (10) and (15) that both $\mathrm{H}$ and $\mathrm{G}$ approach the same equilibrium value. This is equivalent to the steadystate condition:

$$
R_{\infty}=\lambda N_{\infty}
$$

Equation (14) may be simplified by observing the following identities,

$$
\begin{aligned}
\sum_{1}^{\infty} \frac{1}{\mathrm{n}^{2}\left(\mathrm{n}^{2} \pi^{2}+\mu\right)} & =\frac{1}{\mu} \sum_{1}^{\infty} \frac{1}{\mathrm{n}^{2}}-\frac{\pi^{2}}{\mu} \sum_{1}^{\infty} \frac{1}{\mathrm{n}^{2} \pi^{2}+\mu} . \\
& \sum_{1}^{\infty} 1 / \mathrm{n}^{2}=\pi^{2} / 6, \\
\sum_{1}^{\infty} 1 /\left(\mathrm{n}^{2} \pi^{2}+\mu\right) & =(1 / 2)[(1 / \sqrt{\mu}) \operatorname{coth} \sqrt{\mu}-1 / \mu] .
\end{aligned}
$$

Equation (18), which may be somewhat obscure, is given by Whittaker and Watson(9). Substitution into Equation (14) enables us to rewrite the expression for G:

$$
\mathrm{G}=3\left(\frac{\operatorname{coth} \sqrt{\mu}}{\sqrt{\mu}}-\frac{1}{\mu}\right)-\exp (-\mu \tau)+\frac{6 \mu \exp (-\mu \tau)}{\pi^{2}} \sum_{1}^{\infty} \frac{\exp \left(-\mathrm{n}^{2} \pi^{2} \tau\right)}{\mathrm{n}^{2}\left(\mathrm{n}^{2} \pi^{2}+\mu\right)} .
$$

If a given isotope is stable, it is convenient to describe the accumulation in terms of the ratio of the amount released to the amount produced. This fraction is calculated by evaluating $G / \lambda t$ in the limit when $\lambda$ and $\mu$ vanish. We find, then, that

$$
\tilde{\mathrm{F}}=\frac{\tilde{N}}{\mathrm{Bt}}=1-\frac{1}{15 \tau}+\frac{6}{\pi^{4} \tau} \sum_{1}^{\infty} \frac{\exp \left(-\mathrm{n}^{2} \pi^{2} \tau\right)}{\mathrm{n}^{4}}
$$

as has also been shown by Booth (3). At equilibrium,

$$
\tilde{F}_{\infty}=1 \text {. }
$$

We should point out that we have used two different nomenclatures in describing the accumulation. The definition of $F$ is fairly natural. It represents the ratio of the number of external atoms to the number produced. This definition is meaningful for a stable isotope. However, if the same definition were applied to a decaying species, F would become vanishingly small as t becomes large, since the number of external atoms approaches a finite limit while the number produced increases without bound. We may, however, generalize $\mathrm{F}$ to be the instantaneous ratio of the nondecayed external atoms to the number of nondecayed atoms in the system. This more general definition is

$$
F=\frac{N}{(B / \lambda)[1-\exp (-\lambda t)]}
$$


for unstable species. This formula reduces to the definition of $\tilde{F}$ as $\lambda$ approaches zero. The parameter G, however, is more convenient to use for evaluating the accumulation. $G$ does have a certain physical significance, moreover. It is the ratio of the number of external nondecayed atoms at any instant to the total number of nondecayed atoms in the system at equilibrium. The two parameters are related by

$$
F=\frac{G}{1-\exp (-\mu T)} \text {. }
$$

When $\mu \tau$ (i. e., $\lambda t$ ) is very small compared to unity, G may be obtained from $\tilde{F}$ by using the approximation

$$
\mathrm{G}=\lambda \mathrm{t} \tilde{\mathrm{F}}
$$

The stable-isotope-release formula, Equation (20), is therefore adequate for the evaluation of the accumulated release when $\mu \tau$ is small, even though the given isotope may be radioactive.

\section{Short-Time Approximations}

The above formulas are ordinarily adequate for the evaluation of release at long times. For very short times, on the other hand, the convergence of the various series is unsatisfactory. In the Appendix we will derive approximations that approach the exact relationships asymptotically for small $\tau$ and which give good accuracy up to $\tau=(-1)$. These approximations provide short-time-release formulas needed for the region in which the equations given above are unsatisfactory.

For short times we find that the release rate and the accumulation are given by

$$
\mathrm{H}=3\{(1 / \sqrt{\mu}) \operatorname{erf} \sqrt{\mu \tau}-(1 / \mu)[1-\exp (-\mu \tau)]\},
$$

and

$$
G=3\{(1 / \sqrt{\mu})[\operatorname{erf} \sqrt{\mu \tau}-2 \sqrt{\mu \tau / \pi} \exp (-\mu \tau)]-(1 / \mu)[1-(1+\mu \tau) \exp (-\mu \tau)]\}
$$

respectively, where the error function is defined by

$$
\operatorname{erf} x=\frac{2}{\sqrt{\pi}} \int_{0}^{x} e^{-x^{2}} d x
$$

In general, Equations (25) and (26) are invalid for $\tau>(-1)$. However, as $\tau$ approaches infinity the short-time values of $H$ and $G$ approach the limit

$$
\mathrm{H}=\mathrm{G}=3(1 / \sqrt{\mu}-1 / \mu) \text {. }
$$

This is precisely the limiting value that the exact $\mathrm{H}_{\infty}$ and $\mathrm{G}_{\infty}$ [Equations (10) and (15)] approach for large $\mu$, since coth $\sqrt{\mu}$ goes to unity as $\mu$ gets large. Moreover, if $\mu$ is large enough, the short-time expressions reach the limiting value of Equation (28) before $\tau=(-1)$. Therefore, for large values of $\mu$, the short-time formulas are generally valid for all $\tau$. 
For stable isotopes, Equations (25) and (26) reduce in the limit to

and

$$
\tilde{\mathrm{H}}=6 \sqrt{\tau / \pi}-3 \tau \text {, }
$$

$$
\widetilde{F}=4 \sqrt{T / \pi}-1.5 \tau \text {. }
$$

Equation (30) has been given previously by Booth (3). The approximations are quite accurate for $\tau \leqslant(-1)$ and thus complement the exact expressions where the latter are difficult to use.

\section{APPLICATION TO REACTOR CONDITIONS}

\section{Numerical Evaluation of Fission-Product Release}

The various formulas which have been developed to describe fission-product release involve several transcendental functions. These functions have been tabulated in several sources. (10-12) Outside of certain prescribed ranges of the argument, however, tabulated values are not always avallable. In many cases, then, it will be convenient to refer to the series representations of these functions. The use of series expansions for the functions will, in addition, enable us to make further simplifications of some of the release formulas which will be valid for certain ranges.

For convenience, we will list the infinite series which we need. An exact series, useful for small $\mu T^{*}$, is

$$
\operatorname{erf} \sqrt{\mu \tau}=2 \sqrt{\frac{\mu \tau}{\pi}} \sum_{n=0}^{\infty} \frac{(-1)^{n}(\mu \tau)^{n}}{n !(2 n+1)}
$$

A semiconvergent approximate series, useful for large $\mu \tau$, and which is in error by not more than the last term used, is

$$
\operatorname{erf} \sqrt{\mu \tau}=1-\frac{\exp (-\mu \tau)}{\sqrt{\pi \mu \tau}} \sum_{n=0}^{\infty} \frac{(-1)^{n}(2 n) !}{n !(4 \mu \tau)^{n}} .
$$

Exact for $\mu<\pi^{2}$, a series useful for small $\mu$ is

$$
\operatorname{coth} \sqrt{\mu}=\frac{1}{\sqrt{\mu}}-\sum_{n=1}^{\infty} \frac{(-1)^{n} 2^{2 n} B_{n} \mu^{(2 n-1) / 2}}{(2 n) !},
$$

\footnotetext{
* Since the various parameters which determine fission-product release are never negative, statements about the valldity or ucefulnes of the formulas will implicitly include the ascumption that the arguments are not negative.
} 
where the $B_{n}$ 's are Bernoulli's numbers (13). The first few terms of the expansion of Equation (33) are

$$
\operatorname{coth} \sqrt{\mu}=\frac{1}{\sqrt{\mu}}+\frac{\sqrt{\mu}}{3}-\frac{\mu^{3 / 2}}{45}+\frac{2 \mu^{5 / 2}}{945}-\frac{\mu^{7 / 2}}{4725}+\frac{2 \mu^{9 / 2}}{93,555}-\ldots
$$

For large $\mu$, the useful expansion is

$$
\operatorname{coth} \sqrt{\mu}=1+2 \sum_{n=1}^{\infty} \exp (-2 \mathrm{n} \sqrt{\mu}) .
$$

The exponential series, which is exact for all values of the argument, is convenient to use where $\mu \tau$ is small:

$$
\exp (-\mu \tau)=\sum_{n=0}^{\infty} \frac{(-1)^{n}(\mu \tau)^{n}}{n !}
$$

It will be convenient at times to work with certain combinations of series. This will eliminate numerical difficulties which arise from taking small differences, and will also give some insight into the behavior of the formulas. Combining Equations (31) and (36) we find

$$
\frac{1}{\sqrt{\mu}} \operatorname{erf} \sqrt{\mu \tau}-2 \sqrt{\frac{\tau}{\pi}} \exp (-\mu \tau)=4 \sqrt{\frac{\tau}{\pi}} \sum_{n=0}^{\infty} \frac{(-1)^{\mathrm{n}}(\mu \tau)^{\mathrm{n}+1}}{(2 \mathrm{n}+3) \mathrm{n} !} .
$$

From Equation (36) we get also

$$
1-(1+\mu \tau) \exp (-\mu \tau)=\sum_{n=2}^{\infty} \frac{(-1)^{n}(n-1)(\mu \tau)^{n}}{n !} .
$$

From Equation (34) we may write

$$
\frac{3 \operatorname{coth} \sqrt{\mu}}{\sqrt{\mu}}-\frac{3}{\mu}=1-\frac{\mu}{15}+\frac{2 \mu^{2}}{315}-\frac{\mu^{3}}{1575}+\frac{2 \mu^{4}}{31,185}-\ldots
$$

It will be convenient when $\mu$ is small and $\mu \tau$ is large, to take the difference between the series expansions of Equations (39) and (36) before evaluating them. This gives

$$
\frac{3 \operatorname{coth} \sqrt{\mu}}{\sqrt{\mu}}-\frac{3}{\mu}-\exp (-\mu \tau)=\left(\tau-\frac{1}{15}\right) \mu-\left(\frac{\tau^{2}}{2 !}-\frac{2}{315}\right) \mu^{2}+\ldots
$$

This formula removes the computational difficulty of taking the difference between two numbers, each of which is very close to unity. 
We may now proceed to evaluate the formulas used to describe fission-product release. In order that the tables cover as wide a range of the parameters as possible, $\mu$ and $\tau$ are taken at powers of ten. Although this practice gives the wanted range, it may occasionally leave gaps in 1 : sted values which introduce more uncertainty than may be desirable. If such is the case, the user may calculate short auxiliary tables to describe the appropriate function in the needed range. However, in most cases it will be possible to use the information in the tables directly or with simple graphical interpolation to examine particular cases.

We consider first the case of a stable isotope. Values of $\tilde{H}$ and $\tilde{F}$ are calculated by using Equations (29), (11), (30), and (20). These results are listed in Table 1, and plotted logarithmically in Figure 1. The values of $\tilde{F}$ have been previously reported(14) but are included here for completeness. For small values of $\tau$, the one-term approximations,

$$
\tilde{\mathrm{H}} \equiv \frac{\tilde{\mathrm{R}}}{\mathrm{B}}=6 \sqrt{\frac{\tau}{\pi}}
$$

and

$$
\tilde{\mathrm{F}} \equiv \frac{\tilde{\mathrm{N}}}{\mathrm{Bt}}=4 \sqrt{\frac{\tau}{\pi}}
$$

are extremely accurate. For sufficiently large values of $\tau$ both $\tilde{\mathrm{H}}$ and $\tilde{\mathrm{F}}$ [Equations (11) and (20)] attain the value unity. This means that the production and diffusion are in equilibrium, and the fission-product concentration in the sphere does not change. The amount remaining in the sphere is a negligible part of all that has been produced.

For unstable isotopes, the evaluation of fission-product release involves the two dimensionless parameters, $\mu$ and $\tau$. After sufficient time, however, equilibrium is reached. This means that both $\mathrm{H}$ and $\mathrm{G}$ attain constant values. Moreover, their equilibrium values are necessarily the same, since the depletion by decay of the external accumulation is precisely balanced by the amount being released. $\mathrm{G}_{\infty}$ and $\mathrm{H}_{\infty}$ are tabulated in Tables 2 and 3, and shown in Figure 2. The insert in Figure 2 enables us to find the equilibrium value to graphical accuracy for any large value of $\mu$. If $\mu$ is small the isotope is relatively stable, whereas large $\mu$ indicates rapid decay. The equilitrium values are accurately approximated by

and

$$
\mathrm{G}_{\infty}=\mathrm{H}_{\infty}=1-\mu / 15
$$

$$
\mathrm{G}_{\infty}=\mathrm{H}_{\infty}=3 / \sqrt{\mu} \text {, }
$$

for very small and very large values of $\mu$, respectively.

Tables 2 and 3 list the transient values of $\mathrm{H}$ and $\mathrm{G}$, respectively. Each of these tables is carried to the point where the functions repeat their values, so the tables may be extended by inspection to any value of the arguments $\mu$ and $\tau$. The tables are calculated using the short-time formulas for $\tau \leqslant(-1)$ and the long-time formulas for $\tau \geqslant(-1)$. As a check on the calculatio' $s$, both formulas are used at the transition value of $\tau$. An occasional small discrepancy occurs in the fifth significant figure, in which case the result of the long-time formula is accepted as correct. 
TABLE 1. RELEASE OF STABLE ISOTOPES

\begin{tabular}{rlr}
\hline & $\widetilde{\mathrm{H}}=\tilde{\mathrm{R}} / \mathrm{B}$ & $\tilde{\mathrm{F}}=\tilde{\mathrm{N}} / \mathrm{Bt}$ \\
\hline$(-12)$ & $3.3851(-6)$ & $2.2568(-6)$ \\
$(-11)$ & $1.0705(-5)$ & $7.1365(-6)$ \\
$(-10)$ & $3.3851(-5)$ & $2.2567(-5)$ \\
$(-9)$ & $1.0704(-4)$ & $7.1363(-5)$ \\
$(-8)$ & $3.3848(-4)$ & $2.2566(-4)$ \\
$(-7)$ & $1.0702(-3)$ & $7.1350(-4)$ \\
$(-6)$ & $3.3821(-3)$ & $2.2553(-3)$ \\
$(-5)$ & $1.0675(-2)$ & $7.1215(-3)$ \\
$(-4)$ & $3.3551(-2)$ & $2.2418(-2)$ \\
$(-3)$ & $1.0405(-1)$ & $6.9865(-2)$ \\
$(-2)$ & $3.0851(-1)$ & $2.1068(-1)$ \\
$(-1)$ & $7.7048(-1)$ & $5.6365(-1)$ \\
$(0)$ & $9.9997(-1)$ & $9.3334(-1)$ \\
$(1)$ & 1.0000 & $9.9333(-1)$ \\
$(2)$ & 1.0000 & $9.9933(-1)$ \\
$(3)$ & 1.0000 & $9.9993(-1)$ \\
$(4)$ & 1.0000 & $9.9999(-1)$ \\
$(5)$ & 1.0000 & 1.0000 \\
\hline \hline
\end{tabular}




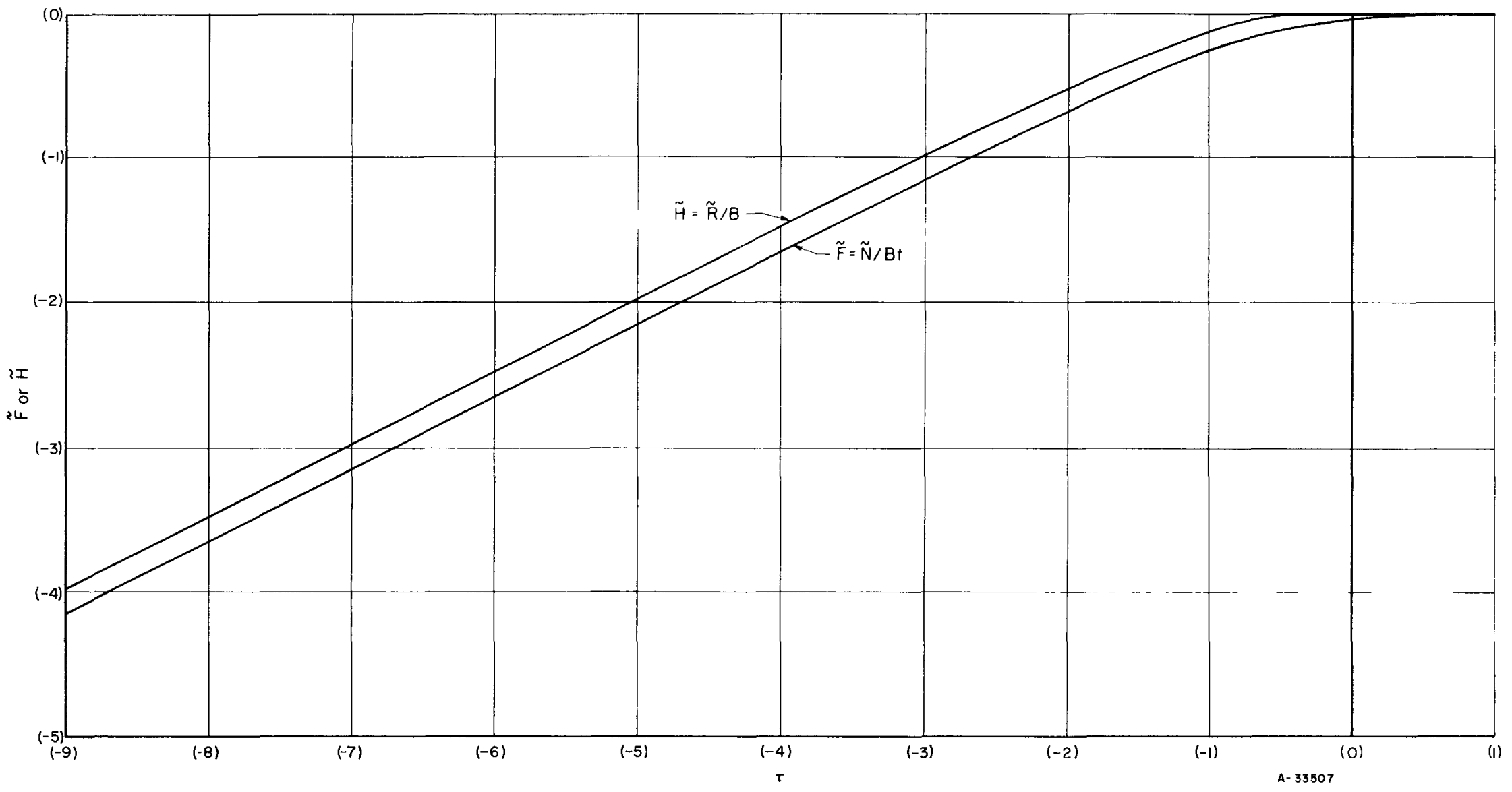

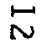

FIGURE 1. RELEASE OF STABLE ISOTOPES 
TABLE 2. RELEASE-RATE FUNCTION, $H=R / B$

\begin{tabular}{|c|c|c|c|c|c|c|c|c|c|c|c|c|c|c|c|c|}
\hline \multirow{2}{*}{$\begin{array}{c}\text { Value of } \\
\mu\end{array}$} & \multicolumn{16}{|c|}{ Release Rate for Value of 7 Shown } \\
\hline & $(-13)$ & $(-12)$ & $(-11)$ & $(-10)$ & $(-9)$ & $(-8)$ & $(-7)$ & $(-6)$ & $(-5)$ & $(-4)$ & $(-3)$ & $(-2)$ & $(-1)$ & $(0)$ & $(1)$ & $\infty$ \\
\hline$(-5)$ & - & - & - & - & - & - & - & - & - & - & - & - & - & $9.9997(-1)$ & 1.0000 & 1.0000 \\
\hline$(-4)$ & - & - & - & - & - & - & - & - & - & - & - & - & $7.7048(-1)$ & $9.9996(-1)$ & $9.9999(-1)$ & $9.9999(-1)$ \\
\hline$(-3)$ & - & - & - & - & - & - & - & - & - & - & - & $3.0851(-1)$ & $7.7046(-1)$ & $9.9990(-1)$ & $9.9993(-1)$ & $9.9993(-1)$ \\
\hline$(-2)$ & - & - & - & - & - & - & - & - & - & - & $1.0405(-1)$ & $3.0850(-1)$ & $7.7027(-1)$ & $9.9930(-1)$ & $9.9933(-1)$ & $9.9933(-1)$ \\
\hline$(-1)$ & - & - & - & - & - & - & - & - & - & $3.3551(-2)$ & $1.0404(-1)$ & $3.0842(-1)$ & $7.6842(-1)$ & $9.9337(-1)$ & $9.9340(-1)$ & $9.9340(-1)$ \\
\hline$(0)$ & - & - & - & - & - & - & - & - & $1.0675(-2)$ & $3.3550(-2)$ & $1.0401(-1)$ & $3.0754(-1)$ & $7.5035(-1)$ & $9.3910(-1)$ & $9.3911(-1)$ & $9.3911(-1)$ \\
\hline (1) & - & - & - & - & - & - & - & $3.3821(-3)$ & $1.0674(-2)$ & $3.3540(-2)$ & $1.0371(-1)$ & $2.9901(-1)$ & $6.0982(-1)$ & $6.5209(-1)$ & - & $6.5209(-1)$ \\
\hline (2) & - & - & - & - & - & - & $1.0702(-3)$ & $3.3820(-3)$ & $1.0671(-2)$ & $3.3440(-2)$ & $1.0073(-1)$ & $2.3385(-1)$ & $2.7000(-1)$ & - & - & $2.7000(-1)$ \\
\hline (3) & - & - & - & - & - & $3.3848(-4)$ & $1.0701(-3)$ & $3.3810(-3)$ & $1.0639(-2)$ & $3.2471(-2)$ & $7.8049(-2)$ & $9.1868(-2)$ & - & - & - & $9.1868(-2)$ \\
\hline (4) & - & - & - & - & $1.0704(-4)$ & $3.3847(-4)$ & $1.0698(-3)$ & $3.370 x(-3)$ & $1.0330(-2)$ & $2.5091(-2)$ & $2.9700(-2)$ & - & - & - & - & $2.9700(-2)$ \\
\hline (5) & - & - & - & $3.3851(-5)$ & $1.0704(-4)$ & $3.3837(-4)$ & $1.0666(-3)$ & $3.2728(-3)$ & $7.9756(-3)$ & $9.4568(-3)$ & - & - & - & - & - & $9.4568(-3)$ \\
\hline (6) & - & - & $1.0705(-5)$ & $3.3850(-5)$ & $1.0701(-4)$ & $3.3736(-4)$ & $1.0356(-3)$ & $2.5262(-3)$ & $2.9970(-3)$ & - & - & - & - & - & - & $2.9970(-3)$ \\
\hline (7) & - & $3.3851(-6)$ & $1.0704(-5)$ & $3.3840(-5)$ & $1.0669(-4)$ & $3.2753(-4)$ & $7.9927(-4)$ & $9.4838(-4)$ & - & - & - & - & - & - & - & $9.4838(-4)$ \\
\hline (8) & $1.0705(-6)$ & $3.3850(-6)$ & $1.0701(-5)$ & $3.3739(-5)$ & $1.0358(-4)$ & $2.5279(-4)$ & $2.9997(-4)$ & - & - & - & - & - & - & - & - & $2.9997(-4)$ \\
\hline (9) & $1.0704(-6)$ & $3.3840(-6)$ & $1.0669(-5)$ & $3.2756(-5)$ & $7.9944(-5)$ & $9.4865(-5)$ & - & - & - & - & - & - & - & - & - & $9.4865(-5)$ \\
\hline (10) & $1.0701(-6)$ & $3.3739(-6)$ & $1.0358(-5)$ & $2.5281(-5)$ & $3.0000(-5)$ & - & - & - & - & - & - & - & - & - & - & $3.0000(-5)$ \\
\hline (11) & $1.0669(-6)$ & $3.2756(-6)$ & $7.9945(-6)$ & $9.4868(-6)$ & - & - & - & - & - & - & - & - & - & - & - & $9.4868(-6)$ \\
\hline (12) & $1.0358(-6)$ & $2.5281(-6)$ & $3.0000(-6)$ & - & - & - & - & - & - & - & - & - & - & - & - & $3.0000(-6)$ \\
\hline (13) & $7.9946(-7)$ & $9.4868(-7)$ & - & - & - & - & - & - & - & - & - & - & - & - & - & $9.4868(-7)$ \\
\hline (14) & $3.0000(-7)$ & - & - & - & - & - & - & - & - & - & - & - & - & - & - & $3.0000(-7)$ \\
\hline
\end{tabular}


TABLE 3 ACCUMULATION FUNCTION, G= NNB

\begin{tabular}{|c|c|c|c|c|c|c|c|c|c|c|c|c|c|c|c|c|c|c|c|c|c|c|}
\hline \multirow{2}{*}{$\begin{array}{l}\text { Value of } \\
\end{array}$} & \multicolumn{22}{|c|}{ Accumulation Function for Value of r Shown } \\
\hline & $(-13)$ & $(-12)$ & $(-11)$ & $(-10)$ & $(-9)$ & $(-8)$ & $(-\pi)$ & $(-6)$ & $(-5)$ & $(-4)$ & $(-3)$ & $(-2)$ & $(-1)$ & (0) & (1) & (2) & (3) & (4) & (5) & (6) & $(T)$ & $\infty$ \\
\hline$(-13)$ & - & - & - & - & - & - & - & - & - & - & - & - & - & - & - & - & - & - & - & - & $10000(-6)$ & 10000 \\
\hline$(-12)$ & - & - & - & - & - & - & - & - & - & - & - & - & - & - & - & - & - & - & - & $10000(-6)$ & $10000(-5)$ & 10000 \\
\hline$(-11)$ & - & - & - & - & - & - & - & - & - & - & - & - & - & - & - & - & - & - & $10000(-6)$ & $99999(-6)$ & $99995(-5)$ & 10000 \\
\hline$(-10)$ & - & - & - & - & - & - & - & - & - & - & - & - & - & - & - & - & - & $99999(-7)$ & $99999(-6)$ & $99995(-5)$ & $99950(-4)$ & 10000 \\
\hline$(-9)$ & - & - & - & - & - & - & - & - & - & - & - & - & - & - & - & - & $99993(-\pi)$ & $99998(-6)$ & $99995(-5)$ & $99950(-4)$ & $99502(-3)$ & 10000 \\
\hline$(-8)$ & - & - & - & - & - & - & - & - & - & - & - & - & - & - & - & $99933(-7)$ & $99993(-6)$ & $99994(-5)$ & $99950(-4)$ & $99502(-3)$ & $95163(-2)$ & 10000 \\
\hline$(-7)$ & - & - & - & - & - & - & - & - & - & - & - & - & - & - & 9 9333(-7) & $99933(-6)$ & $99988(-5)$ & $99949(-4)$ & $99502(-3)$ & $95163(-2)$ & $63212(-1)$ & 10000 \\
\hline$(-6)$ & - & - & - & - & - & - & - & - & - & - & - & - & - & $93334(-7)$ & $99333(-6)$ & $99928(-5)$ & $99943(-4)$ & $99501(-3)$ & $95163(-2)$ & $6.3212(-1)$ & $9995(-1)$ & 10000 \\
\hline$(-5)$ & - & - & - & - & - & - & - & - & - & - & - & - & $56365(-7)$ & $93333(-6)$ & $99328(-5)$ & $99883(-4)$ & $99495(-3)$ & $95162(-2)$ & $63212(-1)$ & $99995(-1)$ & 10000 & 10000 \\
\hline$(-4)$ & - & - & - & - & - & - & - & - & - & - & - & $21058(-7)$ & $56365(-6)$ & $93329(-5)$ & $99283(-4)$ & $99435(-3)$ & $95156(-2)$ & $63211(-1)$ & $99995(-1)$ & $99999(-1)$ & - & $99999(-1)$ \\
\hline$(-3)$ & - & - & - & - & - & - & - & - & - & - & $69865(-8)$ & $21067(-6)$ & $56362(-5)$ & $93284(-4)$ & $98835(-3)$ & $95096(-2)$ & $63205(-1)$ & $99989(-1)$ & $99993(-1)$ & - & - & $99993(-1)$ \\
\hline$(-2)$ & - & - & - & - & - & - & - & - & - & $22418(-8)$ & $69865(-7)$ & $21066(-5)$ & $56333(-4)$ & $92842(-3)$ & $94497(-2)$ & $63145(-1)$ & $99929(-1)$ & $99933(-1)$ & - & - & - & $99933(-1)$ \\
\hline$(-1)$ & - & - & - & - & - & - & - & - & $71215(-9)$ & $22417(-7)$ & $69861(-6)$ & $21005(-4)$ & $56038(-3)$ & $88559(-2)$ & $62552(-1)$ & $99335(-1)$ & $99340(-1)$ & - & - & - & - & $99340(-1)$ \\
\hline (0) & - & - & - & - & - & - & - & $22553(-9)$ & $71215(-8)$ & $22416(-6)$ & $69823(-5)$ & $20943(-3)$ & $53196(-2)$ & $57123(-1)$ & $93906(-1)$ & $93911(-1)$ & - & - & - & - & - & $93911(-1)$ \\
\hline (1) & - & - & - & - & - & - & $71350(-10)$ & $22552(-8)$ & $71211(-\pi)$ & $22404(-5)$ & $69448(-4)$ & $19857(-2)$ & $32638(-1)$ & $65204(-1)$ & $65209(-1)$ & - & - & - & - & - & - & $65209(-1)$ \\
\hline (2) & - & - & - & - & - & $22566(-10)$ & $71350(-9)$ & $22551(-\pi)$ & $71172(-6)$ & $22284(-4)$ & $65828(-3)$ & $12035(-1)$ & $26997(-1)$ & $27000(-1)$ & - & - & - & - & - & - & - & $27000(-1)$ \\
\hline (3) & - & - & - & - & $71363(-11)$ & $22566(-9)$ & $71346(-8)$ & $22539(-6)$ & $70789(-5)$ & $21120(-3)$ & $39772(-2)$ & $91854(-2)$ & $91868(-2)$ & - & - & - & - & - & - & - & - & $91868(-2)$ \\
\hline (4) & - & - & - & $22567(-11)$ & $71363(-10)$ & $22565(-8)$ & $71307(-7)$ & $22418(-5)$ & $67092(-4)$ & $12749(-2)$ & $29695(-2)$ & $29700(-2)$ & - & - & - & - & - & - & - & - & - & $297004-2)$ \\
\hline (5) & - & - & $71365(-12)$ & $22567(-10)$ & $71359(-9)$ & $22553(-\pi)$ & $70923(-6)$ & $21247(-4)$ & $10486(-3)$ & $94552(-3)$ & $94568(-3)$ & - & - & - & - & - & - & - & - & - & - & $94568(-3)$ \\
\hline (6) & - & $22568(-12)$ & $71364(-11)$ & $22566(-9)$ & $71321(-8)$ & $22431(-6)$ & $67218(-5)$ & $12820(-3)$ & $29965(-3)$ & $29970(-3)$ & - & - & - & - & - & - & - & - & - & - & - & $29970(-3)$ \\
\hline (i) & $71365(-13)$ & $22567(-11)$ & $71361(-10)$ & $22554(-8)$ & $70937(-7)$ & $21259(-5)$ & $40557(-4)$ & $94822(-4)$ & $94838(-4)$ & - & - & - & - & - & - & - & - & - & - & - & - & $94838(-4)$ \\
\hline (8) & $71365(-12)$ & $22566(-10)$ & $71322(-9)$ & $22433(-7)$ & $67231(-6)$ & $12827(-4)$ & $29992(-4)$ & $29997(-4)$ & - & - & - & - & - & - & - & - & - & - & - & - & - & $29997(-4)$ \\
\hline (9) & $71361(-11)$ & $22554(-9)$ & $70938(-8)$ & $21261(-6)$ & $40564(-5)$ & $94849(-5)$ & $94865(-5)$ & - & - & - & - & - & - & - & - & - & - & - & - & - & - & $94865(-5)$ \\
\hline (10) & $71322(-10)$ & $22433(-8)$ & $67232(-7)$ & $12828(-5)$ & $29995(-5)$ & $30000(-5)$ & - & - & - & - & - & - & - & - & - & - & - & - & - & - & - & $30000(-5)$ \\
\hline (11) & $70938(-9)$ & $21261(-\pi)$ & $40565(-6)$ & $94852(-6)$ & $94868(-6)$ & - & - & - & - & - & - & - & - & - & - & - & - & - & - & - & - & $94868(-6)$ \\
\hline (12) & $67232(-8)$ & $12828(-6)$ & $29995(-6)$ & $30000(-6)$ & - & - & - & - & - & - & - & - & - & - & - & - & - & - & - & - & - & $30000(-6)$ \\
\hline (13) & $40565(-7)$ & $94852(-7)$ & $94868(-7)$ & - & - & - & - & - & - & - & - & - & - & - & - & - & - & - & - & - & - & $948684-\pi$ \\
\hline (14) & $29995(-7)$ & $30000(-7)$ & - & - & - & - & - & - & - & - & - & - & - & - & - & - & - & - & - & - & - & $30000(-7)$ \\
\hline (15) & $94868(-8)$ & - & - & - & - & - & - & - & - & - & - & - & - & - & - & - & - & - & - & - & - & $94868(-8)$ \\
\hline
\end{tabular}




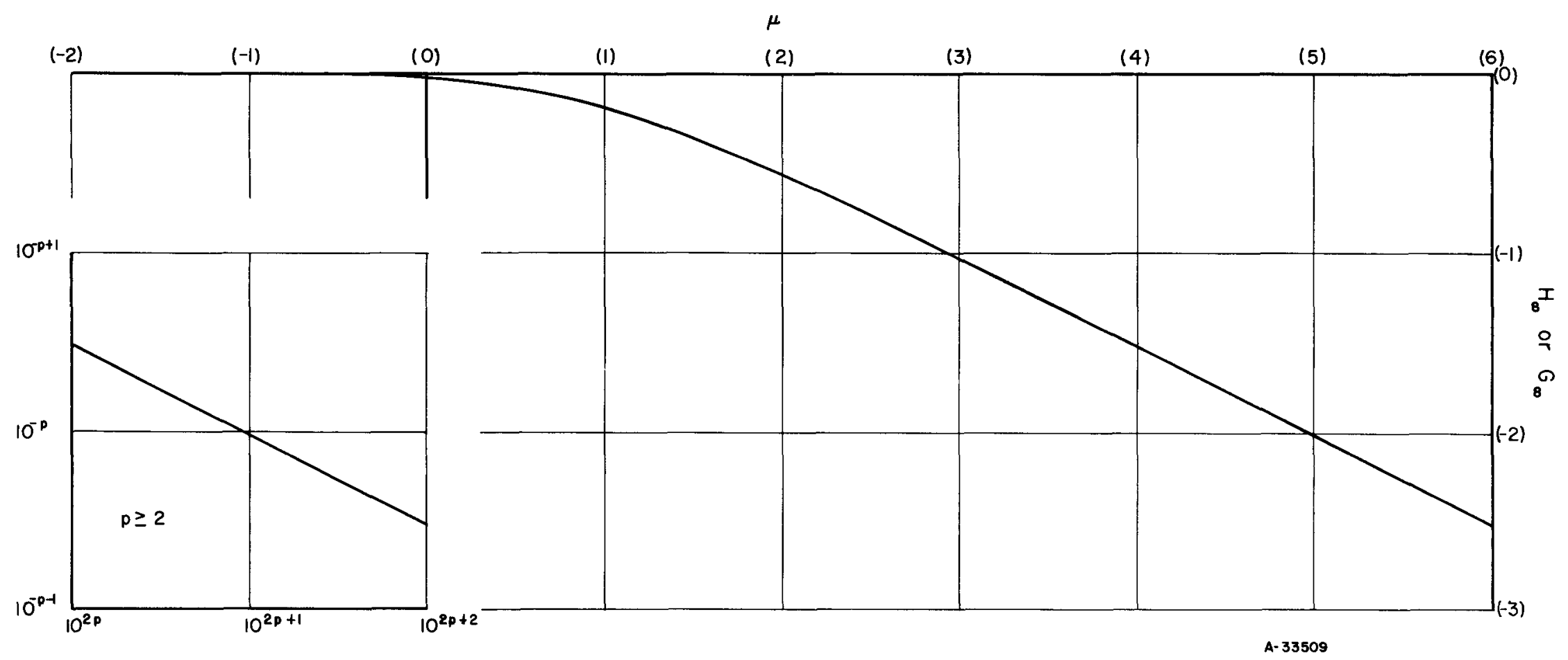

FIGURE 2. SIEADY-STATE RELEASE RATE ANE ACCUMULATION FUNCTIONS 
In using the short-time formulas, we take advantage of the fact that we have only functions of $\mu \mathrm{T}$ multiplied by $1 / \sqrt{\mu}$ or $1 / \mu$. This enables us to calculate these functions just once for a given $\mu \mathrm{T}$ diagonal. We then take linear combinations of the se functions to obtain the desired entries. The needed functions themselves are obtained from Equations (31), (32), (36), (37), or (38), or they are obtained from standard mathematical tables of the error and the exponential functions.

In the long-time formulas, Equations (9) and (19), the series portion makes no contribution for $\tau \geqslant 10$. Equations (39) and (40) are useful for evaluating the formulas when $\mu$ is too small to permit use of mathematical tables of hyperbolic functions.

The repetition of values at the extremities of the tables comes about from the structure of the formulas. As $\mu$, $\tau$, or $\mu \tau$ reach certain values, the formulas attain particularly simple forms, so that varying the argument by an order of magnitude causes the function to vary by a constant factor. In the following discussion, we shall designate limiting values of the arguments for which the simple formulas are valid. These limits are chosen such that five-significant-figure accuracy is maintained. However, in many cases, the limiting values of the arguments may be weakened by one or more orders of magnitude, and adequate accuracy will still be maintained.

If $\mu \tau \leqslant(-5)$ or $\mu \leqslant(-5)$, then

$$
\mathrm{H}=\tilde{\mathrm{H}} \text {. }
$$

If $\mu \tau \geqslant(1)$ or $\tau \geqslant(1)$,

$$
\mathrm{H}=\mathrm{H}_{\infty}
$$

If $\tau \leqslant(-12)$,

$$
\mathrm{H}=\frac{3 \operatorname{erf} \sqrt{\mu T}}{\sqrt{\mu}} .
$$

If $\tau \leqslant(-10)$ and $\mu \tau \leqslant(-5)$,

$$
\mathrm{H}=6 \sqrt{\tau / \pi} .
$$

If $\mu \tau \leqslant(-6)$,

$$
\mathrm{G}=\mu T \mathrm{~F}
$$

If $\mu T \geqslant(2)$,

$$
G=G_{\infty}
$$

If $\mu \leqslant(-13)$,

$$
G=\frac{3}{\sqrt{\mu}}\left[\operatorname{erf} \sqrt{\mu \tau}-2 \sqrt{\frac{\mu \tau}{\pi}} \exp (-\mu \tau)\right] .
$$

If $\tau \leqslant(-11)$ and $\mu \tau \leqslant(-6)$,

$$
\mathrm{G}=\frac{4 \mu \tau^{3 / 2}}{\sqrt{\pi}}
$$


If $\tau \geqslant(7)$ and $\mu \leqslant(-5)$,

$$
G=1-\exp (-\mu \tau)
$$

If $\tau \geqslant(7)$ and $\mu \tau \leqslant(-5)$,

$$
\mathrm{G}=\mu \tau \text {. }
$$

Interpretation of Data

An experiment will ordinarily give several data for a given isotope, relating the release rate or accumulation of the isotope to the time. If the conditions of the experiment are sufficiently close to those assumed in the analysis, it is possible to interpret the data to obtain an estimate of the value of $D^{\prime}$ (that is, $D / a^{2}$ ) for that isotope in the given fuel element. We may expect that other fuel elements, made to the same specifications and irradiated at the same temperature, will be characterized by the same valuc of $\mathrm{D}^{-}$for the isotope. The extent to which results may be extrapolated, and indeed, the extent to which fission-product release is actually described by the model used in this report, are questions which must be answered by a combination of extensive experimentation and sound technical judgment.

We expect that ordinarily our data will consist of values of $R$ or $N$ for a given isotope at various times. These data are first transformed into their nondimensional equivalents, H or G. Since $\mathrm{D}^{\prime}$ is as yet unknown, we cannot transform to $\tau$. The data may then be plotted, and compared with curves of $H$ or $G$ versus $t$ for the appropriate value of $\lambda$ and for various values of $\mathrm{D}^{\prime}$. We then find by inspection or interpolation the value of $D^{\prime}$ for the curve which best fits the data.

To illustrate the method of constructing these level curves let us take as an example an isotope for which $\lambda=5(-4)$. A given row in the $H$ (or $G$ ) table corresponds to a particular value of $\mathrm{D}^{\prime}$, since

$$
\mathrm{D}^{\prime}=\lambda / \mu \text {. }
$$

Thus $\mu=(10)$ corresponds to $D^{\prime}=5(-14) ; \mu=(9)$ corresponds to $D^{\prime}=5(-13)$; etc. A given $\tau$ will then correspond to a different value of t for each value of $\mu$. Thus for $\mu=$ (10) and $\tau=(-13), \mathrm{t}=2(0)$. The value of $\mathrm{H}$ from Table 3 then corresponds to $D^{\prime}=$

\begin{tabular}{|c|c|}
\hline$D^{\prime}$ & $\mu$ \\
\hline $5(-16)$ & (12) \\
\hline $5(-15)$ & (11) \\
\hline $5(-14)$ & (10) \\
\hline $5(-13)$ & (9) \\
\hline $5(-12)$ & (8) \\
\hline $5(-11)$ & (7) \\
\hline
\end{tabular}
$5(-14)$ and $t=2(0)$. We may illustrate these relationships with a brief tabulation.

$H$ for $\lambda=5(-4)$ and Indicated Value of $t$

\begin{tabular}{l}
$t=2(0)$ \\
\hline $1.0701(-7)$ \\
$3.3840(-7)$ \\
$1.0701(-6)$ \\
$3.3840(-6)$ \\
$1.0701(-5)$ \\
$3.3840(-5)$
\end{tabular}

$t=2(1)$
$3.3739(-7)$
$1.0669(-6)$
$3.3739(-6)$
$1.0669(-5)$
$3.3739(-5)$
$1.0669(-4)$

$\frac{t=2(2)}{1.0358(-6)}$
$3.2756(-6)$
$1.0358(-5)$
$3.2756(-5)$
$1.0358(-4)$
$3.2753(-4)$

$t=2(3)$
$2.5281(-6)$
$7.9945(-6)$
$2.5281(-5)$
$7.9944(-5)$
$2.5279(-4)$
$7.9927(-4)$
$t=2(4)$ to $\infty$ $3.0000(-6)$

9. $4868(-6)$

$3.0000(-5)$

9. $4865(-5)$

2. $9997(-4)$

9. $4838(-4)$ 
A tabulation like this may be constructed for $\mathrm{H}$ or $\mathrm{G}$ in any region of interest and for any value of $\lambda$. We may then use the information from the table to plot the family of curves of $H$ or $G$ versus t. Figures 3 and 4 show families of curves of $H$ and $G$ versus $t$ for $\lambda=5(-4)$ and for various values of $D^{\prime}$. These curves are plotted logarithmically.

Experimental data for an isotope for which $\lambda=5(-4)$ may be plotted on the graph which contains the appropriate family of curves. Presumably we would be able to fit a curve of the family to these data. We may find the $D^{\prime}$ associated with the experimental curve by logarithmic interpolation. Similar interpolation may be performed to construct the curve for a value of $\mathrm{D}^{\prime}$ in between two members which have been plotted. For the determination of $D^{\prime}$ we will work with discrete points on the experimental curve.

Logarithmic interpolation may be performed either numerically or graphically. If done numerically, each number involved must be converted to its logarithm. The interpolation is performed between the numerical values of the logarithms, and the result converted back to the appropriate natural number.

There are two ways to interpolate graphically. Each requires a scale which is graduated logarithmically and which is separate from the graph. (A section of logarithmic graph paper will do nicely for a scale.) Let us assume we are trying to find the value of $D^{\prime}$ for an experimental point lying between two members of the family of calculated curves. Designate the se as $D_{1}^{\prime}$ and $D_{2}$. We then label the scale with $D_{1}$ and $\mathrm{D}_{2}^{\prime}$. Each point on the scale now represents a value of $\mathrm{D}^{\prime}$. Designate the coordinates of the experimental point on the graph as $\left(t_{0}, \mathrm{H}_{0}\right)$.

The first method is the most general. Define the points $\left(t_{0}, H_{1}\right)$ and $\left(t_{0}, H_{2}\right)$ as the intersections of the vertical line through $\left(t_{0}, H_{0}\right)$ with the $D_{1}^{\prime}$ and the $D_{2}$ curves, respectively.

Draw horizontal lines on the graph through $\left(t_{0}, \mathrm{H}_{1}\right)$ and $\left(t_{0}, \mathrm{H}_{2}\right)$. Lay the scale on the graph so that $D_{1}^{\prime}$ of the scale is on the horizontal line through $\left(t_{0}, H_{1}\right)$ and the $D_{2}$ of the scale on the horizontal line through $\left(t_{0}, \mathrm{H}_{2}\right)$. The scale will be oriented obliquely with respect to the graph. Now slide the scale horizontally until the edge of the scale crosses the experimental point $\left(t_{0}, H_{0}\right)$. The value of $D^{\prime}$ on the scale at this point is the value of $D^{\prime}$ for the curve of the family which passes through the point.

The second method is simpler and faster. However, it is restricted to regions in which the members of the family of curves are parallel straight lines. In this method, we lay the scale on the graph with $D_{j}$ of the scale on the $D_{i}$ curve of the graph and with $\mathrm{D}_{2}$ of the scale on the $\mathrm{D}_{2}^{\prime}$ curve. We now slide the scale along the curves until the edge of the scale crosses the experimental point. We read $\mathrm{D}^{\prime}$ on the scale as before.

If we wish to draw the curve for a given value of $\mathrm{D}^{\prime}$ we use procedures similar to the ones above to locate points on the curve. We will not describe the steps in detail since they should be evident once the interpolation procedures are clear.

There are other ways of interpreting data besides the graphical method described. If we have only a small amount of data, and if $\lambda t$ is small enough or large enough, we may be able to solve the approximation equations analytically for $\mathrm{D}^{\prime}$. This method is more precise than the graphical methods, but the precision may be more than can be justified by the accuracy of the data. For most applications the graphical method of data analysis is sufficiently accurate. 


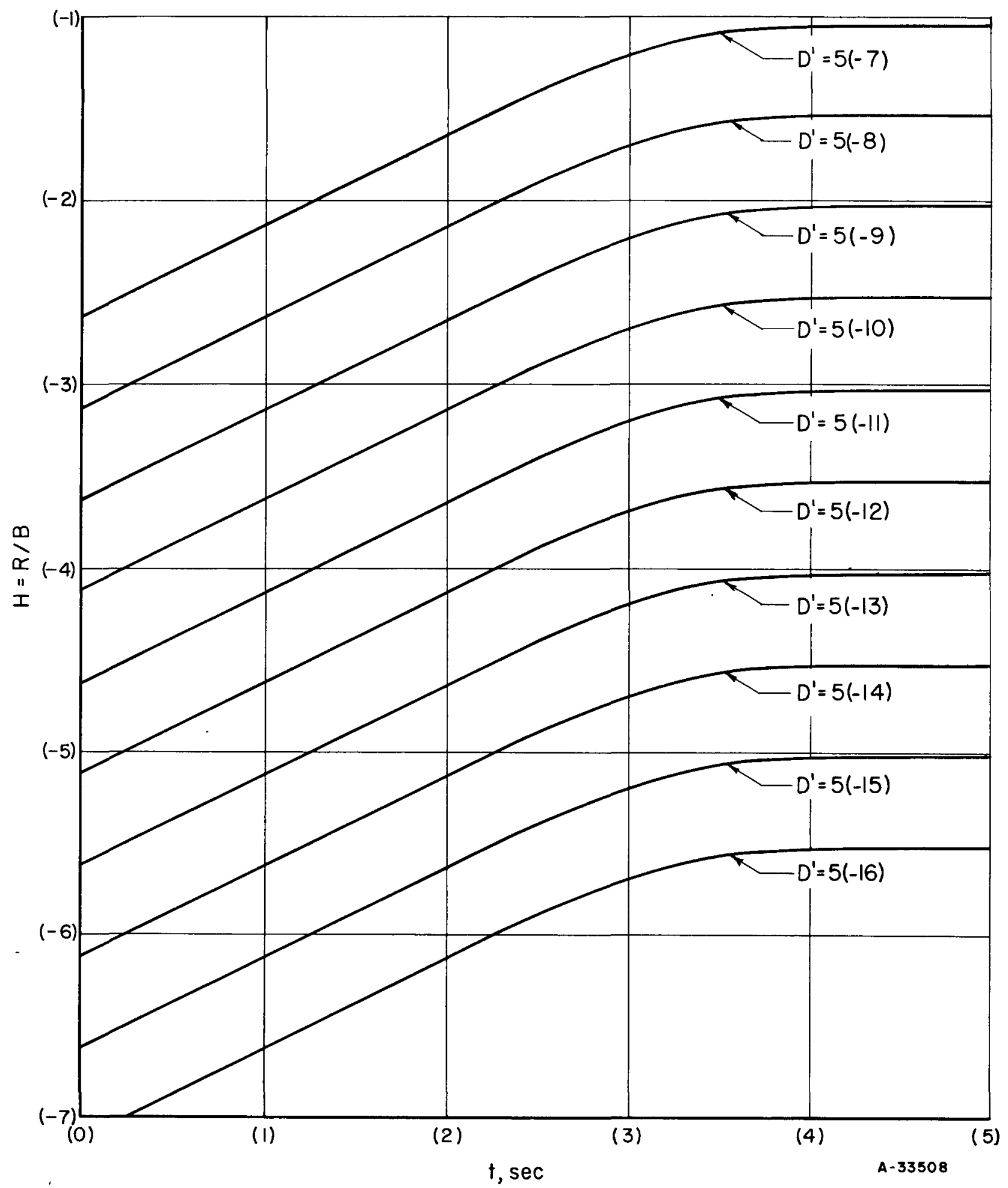

FIGURE 3. RELEASE RATE FOR $\lambda=5(-4) \mathrm{SEC}^{-1}$ 


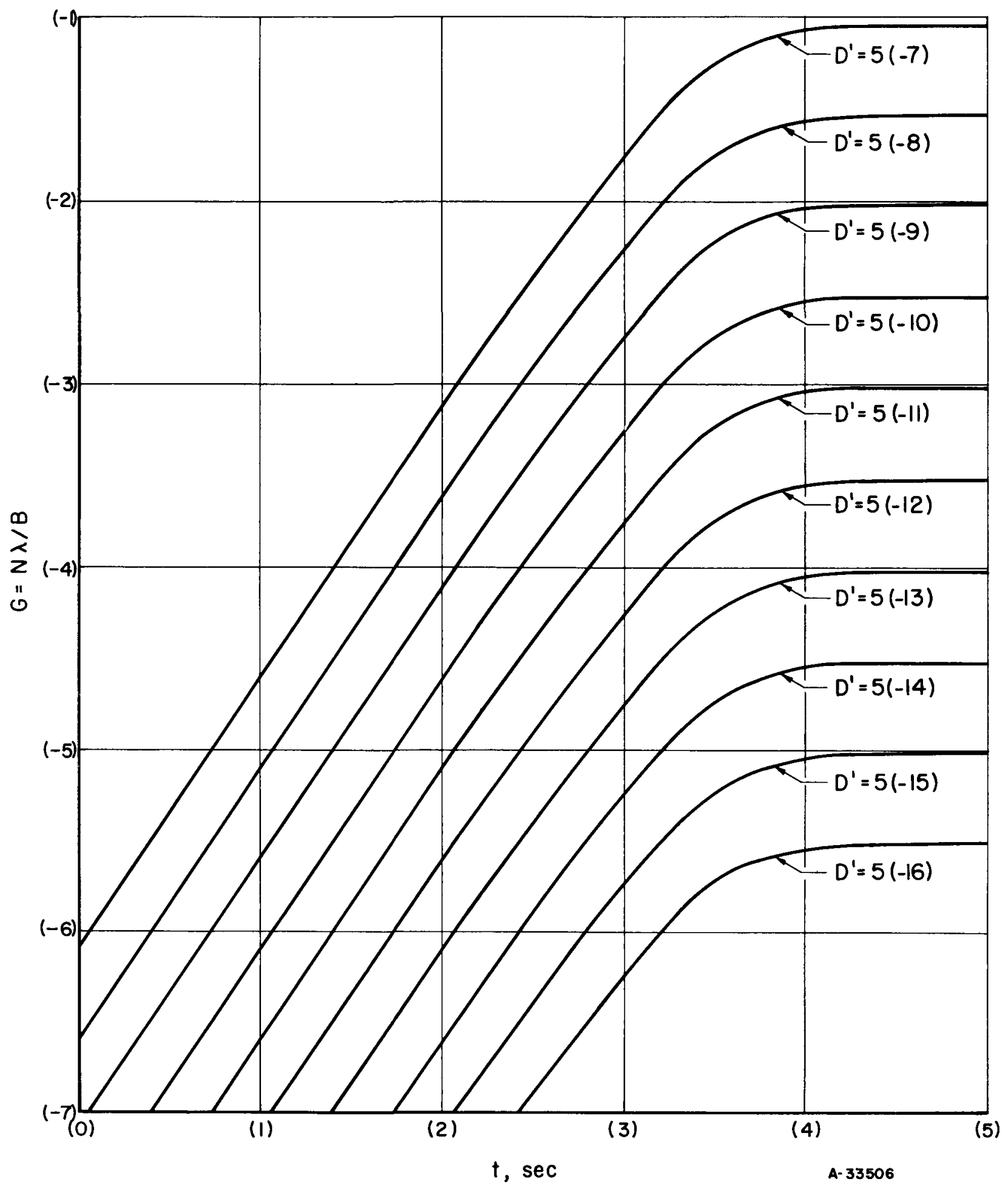

FIGURE 4. EXTERNAL ACCUMULATION FOR $\lambda=5(-4) \mathrm{SEC}^{-1}$ 
This report is concerned with an analysis of fission-product release from porous fuel elements during reactor operation. The analysis is based on diffusion from an equivalent sphere. We are primarily interested in two quantities, the rate of release and the accumulation of fission product which has been released and has not yet decayed. Each of these quantities is referred to a unit volume of the solid.

Mathematical expressions are developed to represent these quantities for any time from the start of irradiation until steady state is achieved. The initial release depends only on the surface area and is equivalent to that from the same amount of surface of a semi-infinite solid. To be more precise, we may say the difference between the release from a sphere and from a semi-infinite solid is less than 1 per cent as long as

$$
\tau \leqslant(-4)
$$

If the half-life of the isotope is sufficiently short, that is, if

$$
\mu \geqslant(5)
$$

steady-state conditions will be reached before $\tau=(-4)$. In other words, if inequality (57) holds, the release is strictly a surface-area phenomenon.

If steady-state conditions have not been reached when $\tau=(-4)$ then the geometry of the particle begins to affect the results. This effect is due to the curvature of the particle surface, and the finite character of the source of fission product.

The equivalent-sphere concept represents an attempt to characterize the fuel material in a manner which is general enough so that the analysis can be extended beyond the point where the surface area dominates the release. Within the limitations imposed by the assumptions we have a broad description of fission-product release from porous fuel materials under reactor conditions.

\section{ACKNOWLEDGMENTS}

The author wishes to express his sincere appreciation to J. C. Bell and L. E. Hulbert for their valuable suggestions and contributions, to S. T. Henderson for performing the computations, and to B. W. Dunnington for his suggestion of the problem and his enthusiastic encouragement during the investigation. 
(1) Cubicciotti, D., "The Diffusion of Xenon From Uranium Carbide-Impregnated Graphite at High Temperatures", NAA-SR-194 (October 13, 1952).

(2) Booth, A. H. , and Rymer, G. T., "Determination of the Diffusion Constant of Fission Xenon in $\mathrm{UO}_{2}$ Crystals and Sintered Compacts", CRDC-720 (August, 1958).

(3) Booth, A. H., "A Method of Calculating Fission Gas Diffusion From $\mathrm{UO}_{2}$ and Its Application to the X-2-f Loop Test", CRDC-721 (September, 1957).

(4) Booth, A. H., "A Method for Calculating Fission Gas Diffusion From UOZ Fuel Elements", AECL DCI-27.

(5) Eichenberg, J. D., Frank, P. W., Kisiel, T. J., Lustman, B., and Vogel, K. H. , "Effects of Irradiation on Bulk $\mathrm{UO}_{2}$ ", WAPD-183 (October, 1957).

(6) Cottrell, W. B., and Culver, H. N., "Machine Calculations for the Diffusion of Fission Products", ORNL CF-59-1-1 (January, 1959).

(7) Crank, J., The Mathematics of Diffusion, Oxford University Press, London (1956), Chapter VI.

(8) Churchill, Ruel V., Fourier Series and Boundary Value Problems, McGraw-Hill Book Company, Inc., New York (1941), pp 102-4.

(9) Whittaker, E. T., and Watson, G. N., A Course of Modern Analysis, Cambridge University Press, Cambridge (1927), p 136.

(10) National Bureau of Standards, Tables of the Error Function and Its Derivative, Washington (1954).

(11) National Bureau of Standards, Tables of the Exponential Function $\mathrm{e}^{\mathrm{x}}$, Washington (1951).

(12) National Bureau of Standards, Tables of Circular and Hyperbolic Sines and Cosines for Radian Arguments, Washington (1939).

(13) Dwight, H. B., Tables of Integrals and Other Mathematical Data, Revised Edition, Macmillan Company, New York (1947), p 10.

(14) Cunningham, G. W., Beck, S. D., and Dunnington, B. W., "Design of Oxide Fuel Elements", Paper 31, Fuel Elements Conference, TID-7559 (Part 1) (August, $1959)$. 
APPENDIX

DERIVATION OF SHORT-TIME-RELEASE FORMULAS 


\section{APPENDIX}

\section{DERIVATION OF SHOR T-TIME-RELEASE FORMULAS}

To develop the short-time formula for the release rate we start with Equation (9),

$$
\mathrm{H}=3\left(\frac{\operatorname{coth} \sqrt{\mu}}{\sqrt{\mu}}-\frac{1}{\mu}\right)-6 \exp (-\mu \tau) \sum_{n=1}^{\infty} \frac{\exp \left(-\mathrm{n}^{2} \pi^{2} \tau\right)}{\mathrm{n}^{2} \pi^{2}+\mu} .
$$

Consider $\mathrm{H}$ as a function of $\tau$. Note that for $\tau=0$,

$$
\mathrm{H}=0 \text {. }
$$

Take the derivative of $\mathrm{H}$ :

$$
\frac{d H}{d \tau}=6 \exp (-\mu \tau) \sum_{n=1}^{\infty} \exp \left(-n^{2} \pi^{2} \tau\right)
$$

Consider the following identity** associated with the theta function:

$$
\sum_{n=1}^{\infty} \cos 2 n \pi v \exp \left(-n^{2} \pi^{2} \tau\right)=-\frac{1}{2}+\frac{1}{2 \sqrt{\pi T}} \sum_{n=-\infty}^{\infty} \exp \left[-\frac{(v+n)^{2}}{\tau}\right] .
$$

Equation (A-4) is true for any values of $v$ and $\tau$. Particularly, if we choose $v=0$, twe may write

$$
\sum_{n=1}^{\infty} \exp \left(-n^{2} \pi^{2} \tau\right)=-\frac{1}{2}+\frac{1}{2 \sqrt{\pi \tau}} \sum_{n=-\infty}^{\infty} \exp \left(-\frac{n^{2}}{\tau}\right)
$$

The series on the right-hand side has equal terms for $\mathrm{n}$ and $-\mathrm{n}$, and one term for $\mathrm{n}=0$. Combining the duplicated terms and writing the zero term gives

$$
\sum_{n=1}^{\infty} \exp \left(-n^{2} \pi^{2} \tau\right)=-\frac{1}{2}+\frac{1}{2 \sqrt{\pi \tau}}+\frac{1}{\sqrt{\pi \tau}} \sum_{n=1}^{\infty} \exp \left(-\frac{n^{2}}{\tau}\right) .
$$

We use Equation $(\mathrm{A}-6)$ to replace the series in Equation (A-3). Then

$$
\begin{gathered}
\frac{d H}{d \tau}=-3 \exp (-\mu \tau)+\frac{3 \exp (-\mu \tau)}{\sqrt{\pi \tau}}+\frac{6 \exp (-\mu \tau)}{\sqrt{\pi \tau}} \sum_{n=1}^{\infty} \exp \left(-\frac{n^{2}}{\tau}\right) \\
H=\int_{0}^{\tau}\left(\frac{d H}{d \tau}\right) d \tau .
\end{gathered}
$$

Mignus, W., and Oberhettinger, F., Formulas and Theorems for the Special Functions of Mathematıcal Physıcs, Chelsea Publishmir Company, New York (1949), p 99. 


$$
H=-\frac{3}{\mu}[1-\exp (-\mu \tau)]+\frac{3}{\sqrt{\mu}} \operatorname{erf} \sqrt{\mu \tau}+E
$$

where

$$
E=\frac{6}{\sqrt{\pi}} \sum_{n=1}^{\infty} \int_{0}^{\tau} \frac{\exp (-\mu \tau) \exp \left(-n^{2} / \tau\right)}{\sqrt{\tau}} \mathrm{d} \tau
$$

We may perform the integration in Equation $(A-10)$, giving the result

$$
E=\frac{3}{\sqrt{\mu}} \sum_{1}^{\infty}\left[\exp (-2 \mathrm{n} \sqrt{\mu}) \operatorname{erfc}\left(\frac{\mathrm{n}}{\sqrt{\tau}}-\sqrt{\mu \tau}\right)-\exp (2 \mathrm{n} \sqrt{\mu}) \operatorname{erfc}\left(\frac{\mathrm{n}}{\sqrt{\tau}}+\sqrt{\mu \tau}\right)\right] . \quad(A-11)
$$

Equation $(A-9)$ is an exact formula for $\mathrm{H}$. We make the approximation by assuming $\mathrm{E}$ is negligibly small for $\tau \leqslant 0.1$. In order to demonstrate that $E$ may indeed be neglected we may place a limit on its value. For this purpose it is more convenient to use Equation (A-10) than the integrated result. Let the upper limit of the integral in Equation $(A-10)$ be $\tau_{0}$. As $\tau$ goes from zero to $\tau_{0}$

$$
\exp \left(-\mathrm{n}^{2} / \tau\right) \leqslant \exp \left(-\mathrm{n}^{2} / \tau_{0}\right)
$$

Then

$$
E \leqslant \frac{6}{\sqrt{\pi}} \sum_{n=1}^{\infty} \exp \left(-\mathrm{n}^{2} / \tau_{0}\right) \int_{0}^{\tau_{0}} \frac{\exp (-\mu \tau)}{\sqrt{\tau}} \mathrm{d} \tau
$$

We drop the subscript after integrating:

$$
E \leqslant \frac{6}{\sqrt{\mu}} \operatorname{erf} \sqrt{\mu \tau} \sum_{n=1}^{\infty} \exp \left(-n^{2} / \tau\right)
$$

We observe that for $\tau$ as large as 0.1 , the first term of the series is only exp $(-10)$. The subsequent terms of the series are negligible compared to the first. That means that the terms dropped are less than about $2 \exp (-10)$ times the error function term retained in Equation (A-9). As $T$ gets smaller, the contribution of $E$ rapidly becomes more negligible.

The actual error in the approximation is much less than the upper limit of inequality (A-14). This is determined from the numerical calculations in which the exact and approximate formulas were evaluated at $\tau=0.1$, and found to be in agreement with only an occasional discrepancy of one or two units in the fifth significant figure.

$G$ is obtained from Equation (A-9) by integration, using Equation (13). Since $H$ is never negative, the approximation becomes more accurate with integration. 